\title{
Innovative Developments in HCI and Future Trends
}

\author{
Mohammad S. Hasan ${ }^{1}$ and Hongnian $\mathrm{Yu}^{2}$ \\ ${ }^{1}$ Faculty of Computing, Engineering and Sciences, Staffordshire University, UK \\ ${ }^{2}$ Faculty of Science and Technology, Bournemouth University, UK
}

\begin{abstract}
The recent developments in technology have made noteworthy positive impacts on the human computer interaction (HCI). It is now possible to interact with computers using voice commands, touchscreen, eye movement, hand gesture etc. This paper compiles some of the innovative HCI progresses in various areas e.g. specialised input/output devices, virtual or augmented reality, wearable technology etc. It also identifies some future research directions.
\end{abstract}

Keywords: HCI, Virtual Reality, Augmented Reality, Haptic Feedback Controller, Smart Home.

\section{Introduction}

The recent technological developments in computing have changed the Human Computer Interaction (HCI) quite significantly. Current desktop or laptop computers are equipped with speedy and vast processing capabilities e.g. multi-core processors with hyper threading, larger and faster main memory, powerful graphics cards, solid state drive (SSD) based secondary memory as well as built-in input-output devices e.g. web cam, sound card etc. These computers normally run operating systems such as Linux, Mac OS, Windows etc. Similarly, mobile devices e.g. smartphones, smart-watch, tablet computers with 8-core processor, 3GB or higher RAM, 32GB or higher SSD storage, multi-touch screen, high resolution cameras, global positioning system (GPS), Near Field Communication (NFC), sensors e.g. proximity, finger print, acceleration, barometer etc. are common nowadays. Many of these advancements have enabled these devices to process information in real time e.g. voice commands, human body pulse rate monitoring etc. Furthermore, the cost of such devices has dropped drastically because of the competitions among the leading manufacturers e.g. Apple, Asus, Dell, HP, Lenovo, Samsung etc. The mobile devices generally run operating systems such as Android, Bada, iOS, Tizen, Windows etc. The mobile computing $\mathrm{HCI}$ has developed in diverse directions and users can interact with modern devices in many ways e.g. touch, voice, heart pulse, body temperature etc.

This paper reviews some of the latest developments in hardware and software for HCI in various areas e.g. specialised input/output devices, virtual or augmented reality, wearable technology etc. It also attempts to identify future trends, research directions and challenges.

The rest of the paper is organised as follows. Sections 2 to 9 discuss some of the recent innovative developments. And section 10 draws some conclusions.

\section{Specialised Input / Output Devices}

\subsection{Curved and Ultra High Definition (UHD) or 4K Resolution Displays}

Curved displays or monitors, shown in Figure 1, are already available as consumer products and are manufactured by Dell, LG, Samsung etc. These offer better viewing angle, less reflection, better 3D experience etc. However, these displays also have some drawbacks e.g. higher cost, wall hanging problem etc. [1]. The UHD or $4 \mathrm{~K}$ resolution displays are able to produce 4 times more than the HD i.e. in the order of 4000 pixels horizontally for clearer and crispier viewing experience as shown in Figure 1. Many manufacturers produce displays that are curved and support UHD resolution.

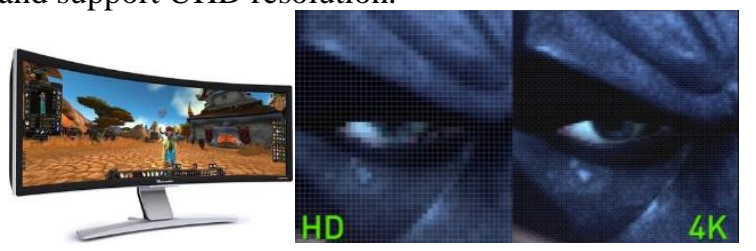

Figure 1: Curved Display and UHD (4K) display [2].

\subsection{Curved and Flexible Displays}

Mobile device manufacturers have launched devices with curved displays e.g. Samsung Galaxy S6 Edge [3] which can display notifications, text messages, weather information etc. on the curved edge for the convenience of the user as shown in Figure 2. Other areas of developments are flexible and transparent displays as shown in Figure 2 and Figure 3 that have been developed by Samsung, LG etc. Many of the flexible displays use polymide film as the backplane. Polyimides are strong, flexible plastics that can achieve high degree of curvature by allowing a much thinner backplane than the conventional plastic [4]. Transparent displays with transmittance of $30 \%$ have already been achieved which is shown in Figure 2. These displays could be useful in advertising, security etc. applications. 


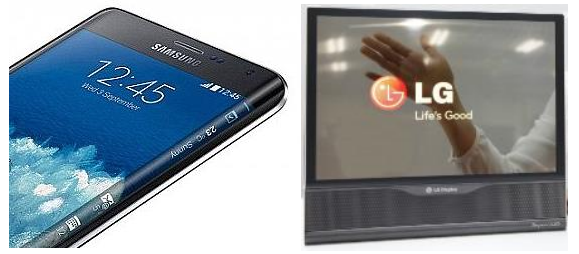

Figure 2: Curved display for smartphone [3] and transparent display [4].

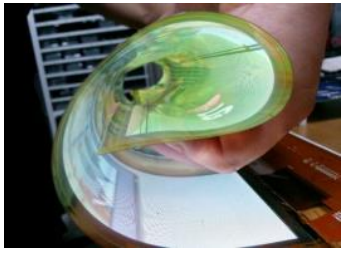

Figure 3: Flexible display [4].

\subsection{Projection or Virtual Keyboard}

It is a small portable device that connects to a smart phone, tablet or a computer e.g. a laptop via a wireless technology e.g. Bluetooth and projects a keyboard on a flat surface to interact with as shown in Figure 4. It allows the user to use a convenient sized keyboard without even carrying a physical keyboard. These devices even take the shape of a key fob. Celluon Epic [5], the V200 key fob [6] etc. are some examples.

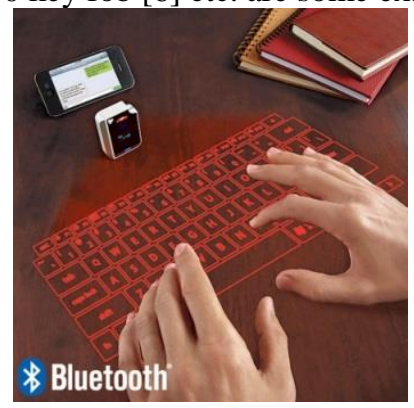

Figure 4: Virtual projection keyboard [6].

\subsection{Hand Gesture, Voice Recognising Devices}

Today daily life devices e.g. smart TV, gaming console etc. integrate HCI support to recognise hand gestures e.g. flip to left or right, move, thumb up, grab, rotation etc. and voice commands etc. And based on the user's interaction, these can perform various actions e.g. menu scrolling, muting, image rotation etc. which is shown in Figure 5. A user can also use one universal device to control multiple devices e.g. TV, audio system etc. Such a device is show in Figure 5 as well. Similarly, modern game consoles and accessories support motion detector, 3D depth sensor etc. and players are able to interact hands free.
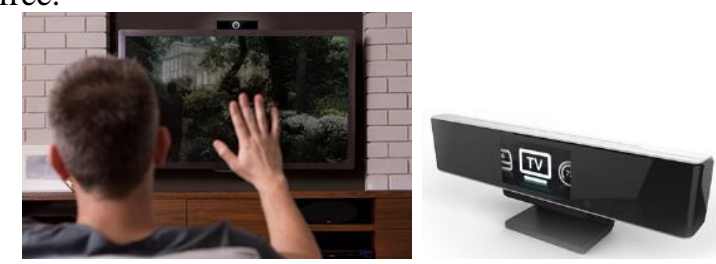

Figure 5: The hand gesture control [7].

\subsection{Brain Signal Capturing Headset}

A brain signal capturing headset is able to detect the changes in voltage when the human brain neurons are working on a thought. The headset normally carries a number of electrodes or sensors that are attached to the human scalp to record the electroencephalographic (EEG) signals and then these signals can be converted into digital form that can be processed by a computer [8]. For example, an epilepsy patient can pick a soft ball using a headset and a robotic arm which is shown in Figure 6. Such systems have been used to drive cars where the driver's captured brain signals are processed by a laptop to drive the vehicle [9]. As an example, the Emotiv EPOC / EPOC+ headset [10] is quite popular in the research community. It offers a convenient brain computer interface with high resolution, 14 EEG channels and 2 references and is shown in Figure 7. The headset has been used to drive a taxi, a wheelchair [11] etc.

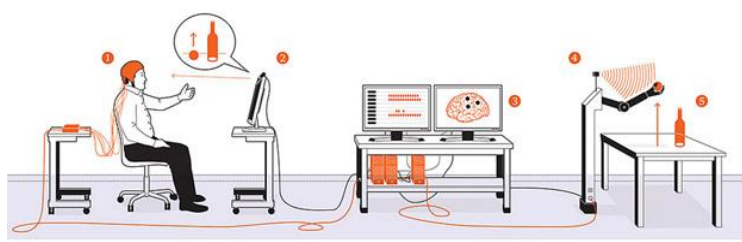

Figure 6: Controlling a robotic arm using a brain signal capturing headset [8].

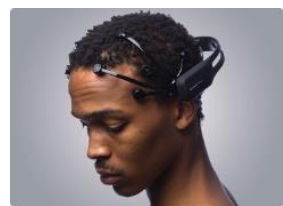

Figure 7: The Emotiv EPOC / EPOC+ headset [10]

\section{Augmented Reality, Virtual Reality, Gaming}

\subsection{Augmented Reality Headset - Microsoft HoloLens}

Microsoft Hololens is an Optical Head-Mounted Display (OHMD) that offers 3D augmented reality platform [12]. It blends holograms with reality where the user is able to design and customise holograms as shown in Figure 8. It is compatible to Windows 10 which is the current version of the Windows operating system.

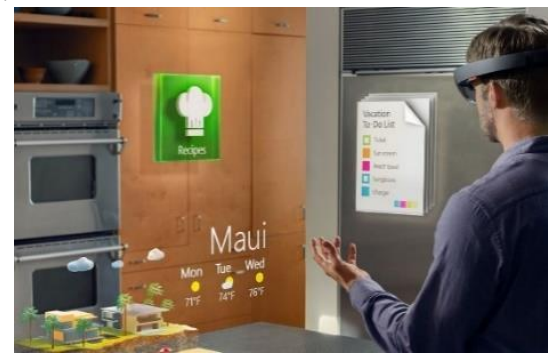

Figure 8: The Microsoft Hololens [12] 


\subsection{Virtual Reality Headset}

Headsets such as HTC Vive, Oculus Rift etc. are very popular in the world of virtual reality, gaming etc. HTC vive carries 70 sensors, 360 degree headtracking with a refresh rate of $90 \mathrm{~Hz}$ to produce lower delay [13]. Oculus Rift [14], shown in Figure 9, offers low latency $360^{\circ}$ head tracking capable of detecting subtle movements to produce natural experience, stereoscopic 3D View etc.

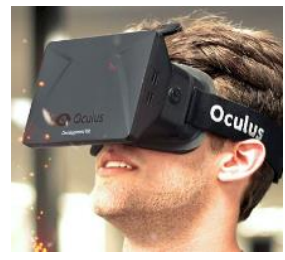

Figure 9: The Oculus Rift Virtual Reality Headset [14].

\subsection{Virtual Mannequin and Virtual Fitting Room}

Many online clothing retailers are now using the "Virtual Mannequin" technology to reduce the volume of returned goods because of wrong size or fitting [15]. The online shoppers enter some of their basic measurements and a virtual mannequin is generated [16] as shown in Figure 10. Another area of development is in-store Virtual Fitting Room. In this case the customer is able to try the clothing virtually in real time and very quickly without even going into a fitting room [17] as shown inFigure 11.

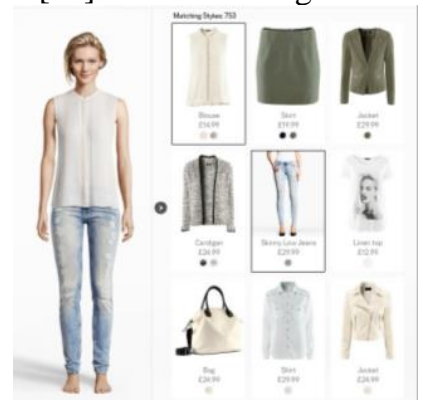

Figure 10: The Virtual Mannequin technology [16]

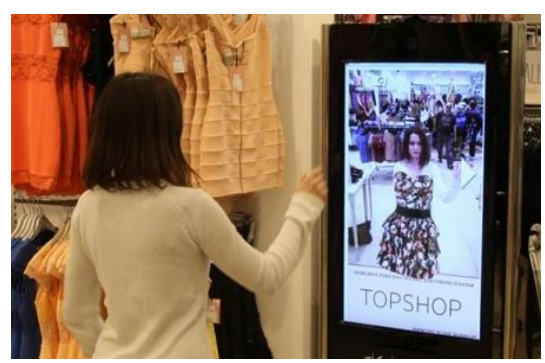

Figure 11: The Virtual Fitting Room [17].

\subsection{Google Cardboard}

Google Cardboard [18] allows users to build a very low cost headset to experience virtual reality using smartphones as shown in Figure 12. As the name suggests, the Google Cardboard comes with cardboard, lenses, straps etc. The smartphone needs to run special application to create the stereoscopic view for both eyes. Various smartphones e.g. Apple iPhone,
Google/LG Nexus, HTC Sensation, Huawei Ascend, LG G2, Optimus, Samsung Galaxy, Sony Xperia etc. are compatible to Google Cardboard.

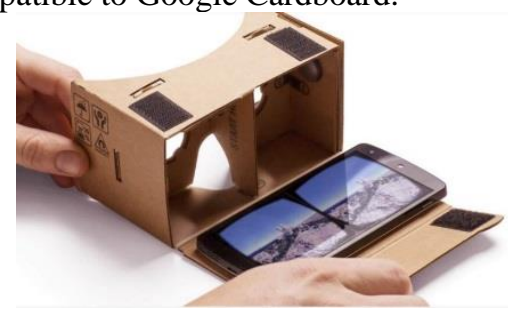

Figure 12: The Google Cardboard [18]

\subsection{Haptic Feedback Controller}

Haptic feedback controllers produce realistic feedback e.g. force, vibration etc. to the user for virtual reality, gaming, tele-robotics, medical applications e.g. computer aided surgery etc. Many current game controllers already support this feature in various forms. For instance, the Steam Controller released by the game developer Valve, shown in Figure 13, offers two track pads to deliver various physical sensations to the player [19]. Another example is Reactive Grip motion controller [20], shown in Figure 13, that can deliver motion and force feedback to the user using sliding contactor plates.

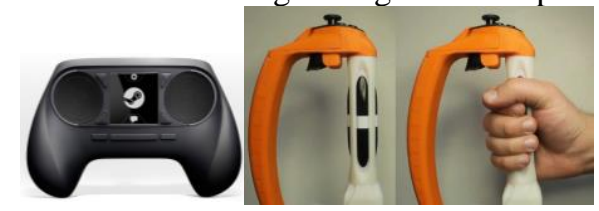

Figure 13: The Steam controller by Valve [19] and The Reactive Grip motion controller [20].

\section{Wearable technology}

\subsection{Smart Glass}

Smart glass e.g. Google Glass is a smart phone like hands free device that is able to take voice commands as shown in Figure 14. It is a heads-up display (HUD) equipped with a camera, microphone and GPS etc. and can perform various tasks e.g. taking and viewing pictures, online searching, reading emails, satellite navigation, taking and making calls etc. [21]. However, Google has stopped commercial production of the Glass in 2015. Another example in this is Garmin's biker's smart glass.

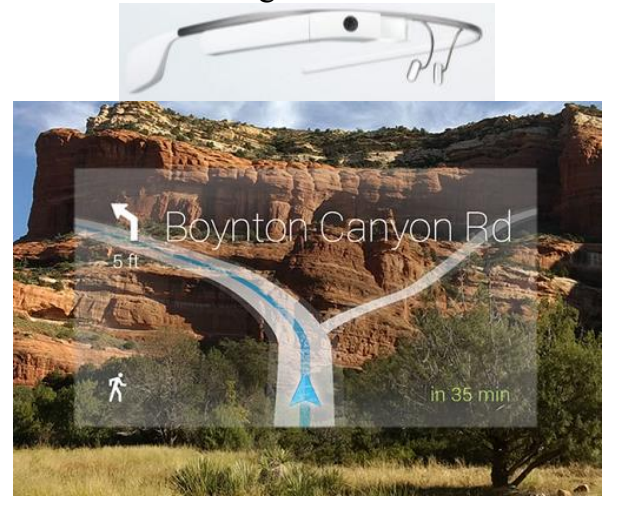


Figure 14: Smart glass e.g. Google glass and its application [21].

\subsection{Smartwatch}

Mobile devices are not limited to only phones, tablet etc. now. Various manufactures e.g. Apple, LG, Motorola, Samsung, Sony etc. have released smart watches as shown in Figure 15. These devices normally communicate with a smartphone using wireless technology e.g. Bluetooth to make or receive phone calls, display messages, notifications etc. Smart watches can also play music, take photos, display stored photos, perform flight check-in, monitor human body fitness, run simple applications etc. without a smartphone [22], [23].

Standalone smartwatches have the functionality of a phone on top of the features offered by a standard smartwatch. These devices allow the user to insert a SIM card into an integrated slot and can connect directly to a $3 \mathrm{G}$ or $4 \mathrm{G}$ cellular network without the need of a phone. The SIM card slot and a typical standalone smartwatch are shown in Figure 16. One major advantage is that these can also be used as a paired device with a smartphone [24].

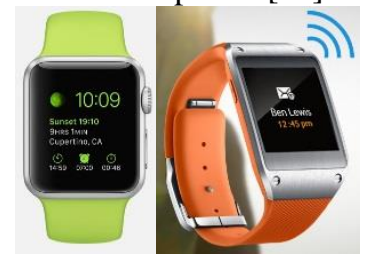

Figure 15: The iWatch by Apple [22] and the Galaxy Gear by Samsung [23]

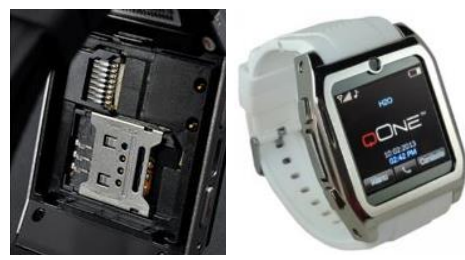

Figure 16: The integrated SIM and micro SD card slots in a standalone smartwatch and QOne Standalone Smartwatch [24]

\subsection{Smart Jewellery}

HCI has also entered the world of fashion and jewellery in the form of Smart devices [25]. For example, the "CSR and Cellini Bluetooth Pendant", shown in Figure 17, is capable of connecting to a smartphone and is able to change its LED colour or brightness depending on the mood or clothing of the user. Furthermore, it can also generate notification of incoming calls, emails or text messages to the user by changing LED colour or flashing or vibrating. Another example is the "June Bracelet or Brooch", also shown in Figure 17, which looks like a diamond in a metal e.g. platinum, gold etc. or on leather strap or it can be worn as a brooch. The device feeds data to a smartphone and shows summary of the user's exposure to the sun. It produces UV index, weather forecast etc. for sunscreen, sunglasses etc. to protect the user's skin from sun damages or premature wrinkles.

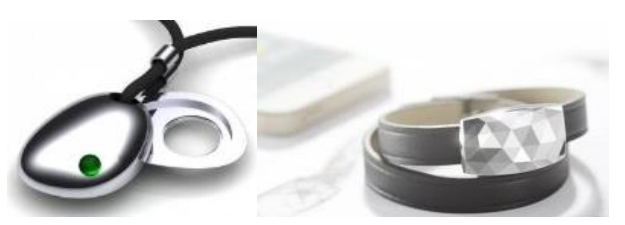

Figure 17: The CSR and Cellini Bluetooth Pendant and the Netatmo June Bracelet or Brooch [25].

\subsection{Smart Footware}

Smart shoe, shown in Figure 18, generates power using two devices - a "shock harvester" and a "swing harvester" which produce power when the heel hits the ground and when the foot is swinging, respectively [26]. The generated power is three to four milliWatt $(\mathrm{mW})$ and can be used to power sensors and an antenna. One of the applications of the shoe is indoor navigation and rescue operation. Similarly Bluetooth insoles, shown in Figure 18, are equipped with a number of sensors, accelerometers etc. and can monitor activity levels, walking health issues, therapy progress [27], communicate with smartphones to give directions using vibrations [28] etc. Another area of development is Smart sock which is shown in Figure 19. The smart sock from Sensoria has embedded sensors and is able to produce feedback on the running techniques via smartphone application for a sportsperson [29].
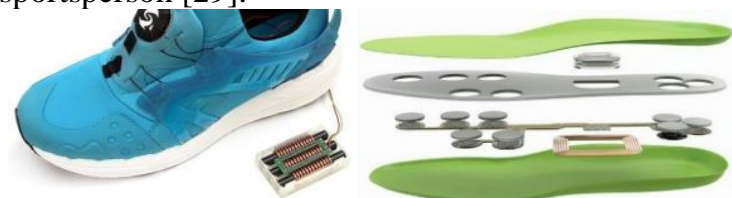

Figure 18: The smart shoe capable of generating power [26] and the Bluetooth insole [27]

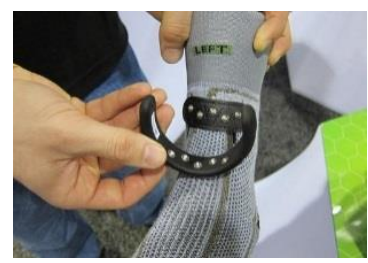

Figure 19: The smart sock from Sensoria Fitness [29].

\section{Fitness, Health, Sports}

\subsection{Fitness Tracking Devices}

A number of fitness tracking devices e.g. Fitbit Charge HR, Fitbit Surge, Basis Peak, Jawbone UP Move, Swarovski Shine etc. are available nowadays [30]. These devices can monitor and display information about the user's heart rate, calorie burning e.g. running, walking, gym activities etc. Some fitness tracking devices e.g. Swarovski Shine, shown in Figure 20, combines the fashion and technology together as these can track running, cycling, swimming etc. activities and can be powered by solar energy. 


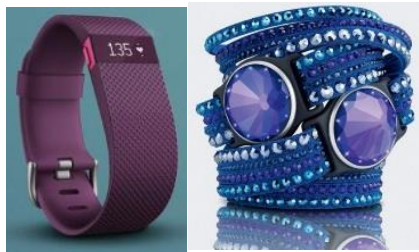

Figure 20: The Fitbit Charge HR and the Swarovski Shine [30].

\subsection{FitGuard}

FitGuard is a mouth guard for athletes which is shown in Figure 21. It contains accelerometers to measure linear and angular acceleration. These sensors can detect activities of the player that exceeds a safety threshold and can generate warning messages to the coach's smart phone or tablet computer. The device also contains LEDs which indicate a potentially low, medium or severe injury of the player with different colours e.g. green, blue, red, respectively [31].

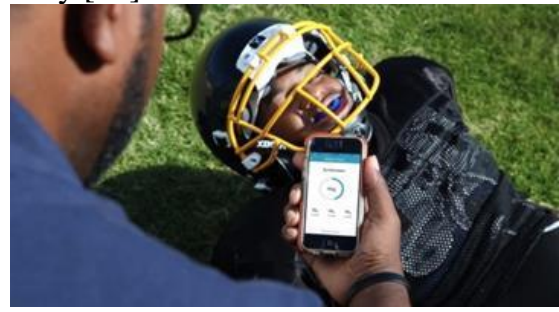

Figure 21: The FitGuard mouth guard for athletes [31].

\subsection{M-Tracer Golf Swing Analyser}

The M-Tracer can eliminate or reduce the cost to hire a golf instructor or trainer to improve a player's performance. It is a small device as shown in Figure 22 , can be attached to the golf club to record many samples of swings during a game. This data then can be sent to a smart phone for further analysis e.g. 3D movement, club speed, angle etc. [32].

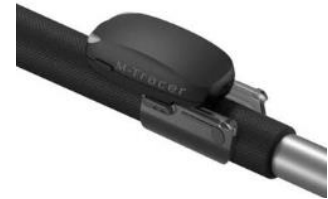

Figure 22: The M-Tracer by Epson [32].

\section{Biomedical, Biometric applications}

\subsection{Smart Contact Lens}

Google has developed a smart contact lens prototype that can measure glucose levels in tears for diabetes patients. The lens is equipped with a tiny wireless chip, a miniaturised glucose sensor and a tiny LED that lights to indicate high glucose level [33], [34] and is shown in Figure 23.

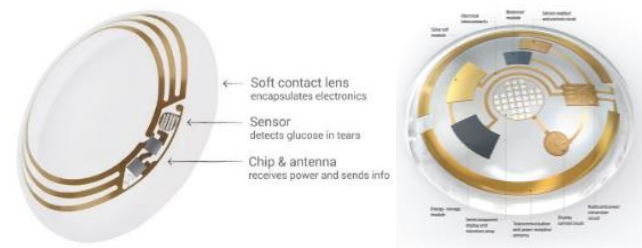

Figure 23: The Google's smart contact lens [33] and the Bionic Contact Lens for augmented reality [35].

\subsection{Bionic Contact Lens}

The bionic contact lens has an antenna to collect incoming radio frequency energy from a separate portable transmitter as shown in Figure 23. Solar energy can also be harvested to provide a boost to the lens. The total collected energy is used to power the internal circuits e.g. display to produce the augmented reality environment, communication with an external computer etc. [35]. The user can read emails, view images e.g. using the contact lens [36].

\subsection{Ultrasound Fingerprint Sensor}

Many mobile devices e.g. smartphones use fingerprint scanner for user authentication. A new type of scanner, shown in Figure 24, has been developed that uses ultrasonic sound waves to scan fingerprints and is able to read finger prints through glass, metal and plastic smartphone covers [37]. It can even scan through sweat, hand lotion, condensation etc.

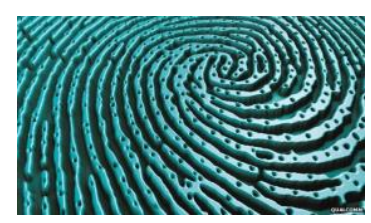

Figure 24: The ultrasound fingerprint sensor revealed by Qualcomm [37].

\subsection{Bio Stamp}

Bio-Stamp which is shown in Figure 25 can be attached to human body skin to measure UV exposure, body temperature, blood pressure etc. [38]. These devices contain flexible circuits and sensors, are powered wirelessly and can stretch like human skin. Bio-Stamps are like temporary tattoos which are able to replace bulky biomedical sensors.

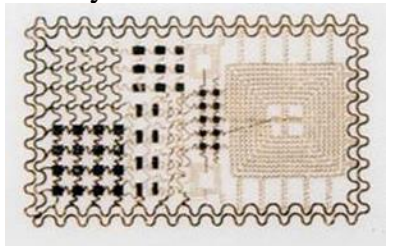

Figure 25: Bio-Stamp [38]

\subsection{Closed Loop Insulin Delivery System}

It is an external autonomous closed loop system which is shown in Figure 26 for diabetic patients. The glucose level of the body is read by a Continuous Glucose Monitor (CGM) and sent to the insulin pump wirelessly. Based on the glucose level, the pump 
injects insulin into the body if needed. Model Predictive or PID Control, Fuzzy logic etc. are used in the control algorithm. Such systems are already manufactured by Medtronic, Animas etc.

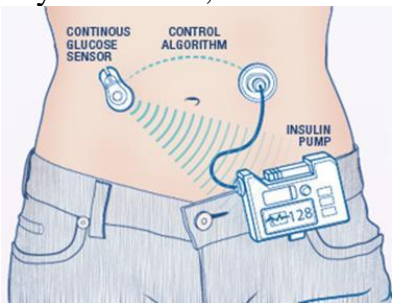

Figure 26: A closed loop insulin delivery system [39].

\subsection{Nervous System or Brain Controlled Robotics}

It is now possible to read the nerve signals of a human body and to control an artificial leg or arm. For example, a patient who lost the lower part of his leg has been fitted with a bionic leg that is controlled by his thoughts [40] which is shown in Figure 27. Another example is brain controlled robotics where the brain signals are used to control a robotic body part e.g. an arm. For example, a patient's body is paralysed from the neck down. Two sensors implanted in his brain can now monitor the brain activities and control a robotic arm. The patient can shake hands of another person, lift a drink, control a computer mouse etc. [41].

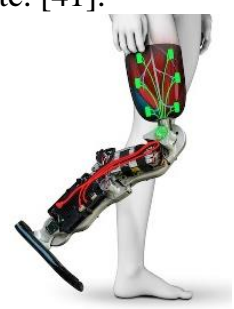

Figure 27: First thought-controlled prosthetic leg [40].

\section{Automobile, Racing}

\subsection{Assisted or Autonomous Driving}

Modern cars have hardware and software to support autonomous maneuvering e.g. auto lane change, active cruise control with lane detection etc. With the auto lane change feature, once the drive activates an indicator for an intended lane change, the car can detect lane marker, other vehicles around it etc. and change the lane when it is safe to do so. A vehicle supporting the active cruise control with lane detection feature can keep a safe distance with the vehicle in front of it while cruising on motorway and produce warning message in case of a lane drift so that the driver can take necessary action to avoid collision with other vehicles. Recently Tesla has launched the "Autopilot" mode for its latest cars where the vehicle can drive itself and can interact with the driver in case of an emergency [42].

\subsection{Automobile Projection System}

Specialised devices e.g. Navdy's transparent HeadUp Display (HUD) [43] are commercially available that can project useful information e.g. navigation, emails, text messages etc. on the windshield of the vehicle as show in Figure 28. These devices connect to a smart phone via wireless technology e.g. Bluetooth and the driver can interact with it using hand gesture, voice commands etc. Such devices ensure safe and legal driving.

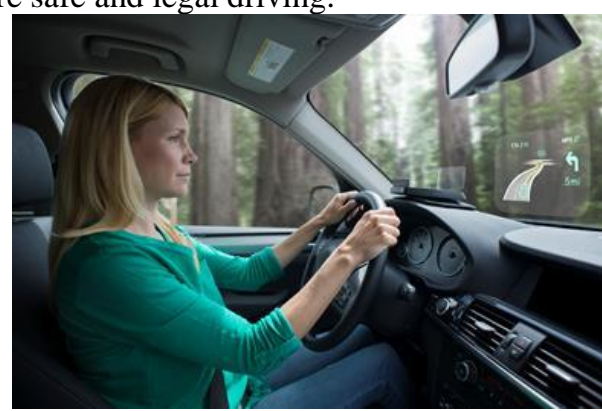

Figure 28: Navdy's transparent Head-Up Display (HUD) [43]

Besides specialised hardware, simple smart phone applications can also produce the projection benefit for the drivers. For example, the HUDWAY smart phone application turns an iPhone or Android device into a projection device as shown in Figure 29. It can improve the driver's safety in low visibility conditions e.g. rain, fog, snow, darkness etc. [44]. The biggest advantage is that such applications do not need any specialised hardware to install.

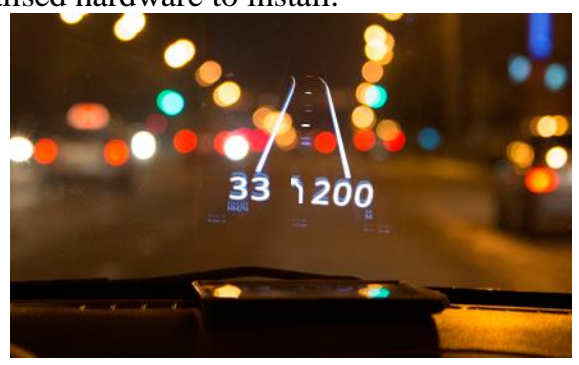

Figure 29: The HUDWAY smart phone application for windshield projection.

\subsection{Safety Suit for Motor Raching}

Accidents in motorcycle racing can cause severe or life threatening injuries to the rider. To ensure the safety, several advanced technology based suits have been developed. For example, the D-Air Racing suit [45] which can be seen in Figure 30, is essentially an intelligent air bag system. It is equipped with accelerometer, gyroscopes, GPS, 2GB of internal memory to monitor the movement of the rider for accidents. In case of a crash, it is able to deploy the airbags in $30 \mathrm{~ms}$. 


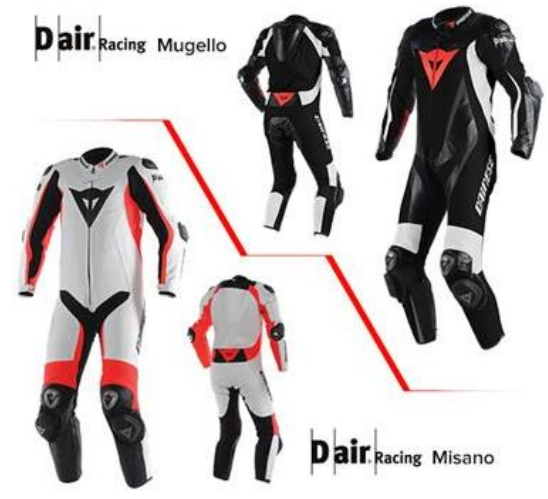

Figure 30: The Dainese D-Air Racing suit [45].

\subsection{Heart Attack Predicting Car Seat}

The car seat which is shown in Figure 31 contains Electrocardiograph (ECG) technology based sensor, camera etc. to detect a heart attack of the driver. The ECG sensors monitor the heart activity through the cloth of the driver. The camera is used to collect the position of the driver. Once a heart attack is detected, the system can take control of the car and bring it to safety autonomously [46].

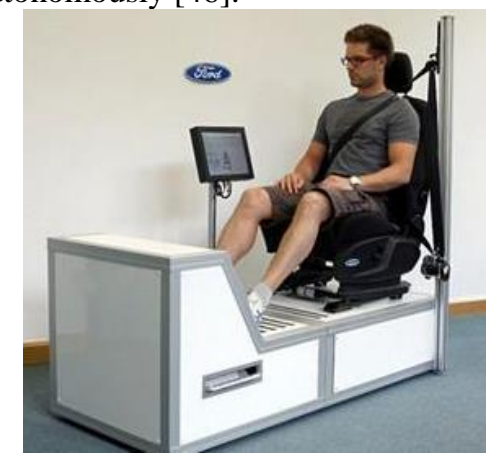

Figure 31: Ford's heart attack detecting car seat [46].

\subsection{Safe Driving Application}

Smart phone applications have been developed to evaluate driving skills of a driver and to generate feedback on journeys for safe driving. Such applications use various sensors e.g. accelerometer, GPS location etc. of the smart phone to record braking, acceleration, speeding etc. habits of the driver. For example, the MotorMate application developed by the car insurance provider Confused.com is shown in Figure 32. The application can help driving to improve their driving style. Furthermore, potential customers can get discount on their car insurance based on the positive feedback produced by the application.

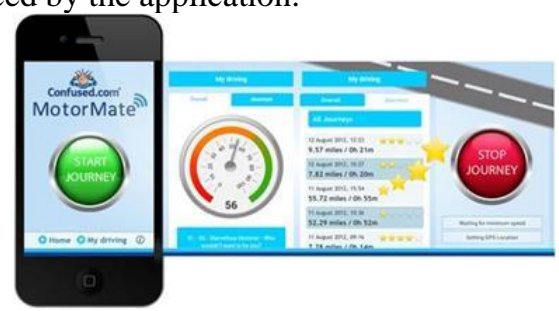

Figure 32: The Confused.com MotorMate smart phone application [47]

\section{Smart Home}

\subsection{Smart Thermostat}

Saving energy has always been one of the prime concerns for a smart home environment. Specialised hardware e.g. the Nest Thermostate, the Hive Thermostat etc., shown in Figure 33, are able to actively monitor the energy use and take action if needed. The Nest Thermostat is able to detect human presence using motion sensors and learn the behavior of the owner. It can be used to control the heating or hot water supply and the user can interact with it directly, using an app or via a web browser [48]. On the other hand, the Hive Thermostat allows the user to interact with it remotely from a phone or via web browser, monitor energy utilization, set and execute heating schedule etc. However, this device does not support any learning capability.

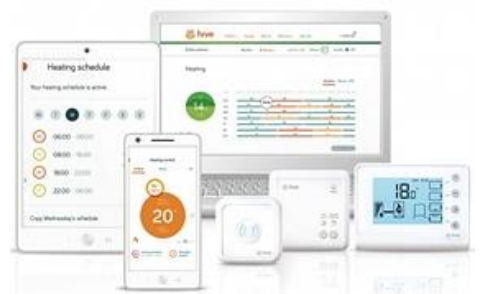

Figure 33: The Nest Thermostat and the Hive Thermostat [48].

\subsection{Smart Bulbs}

LED based light bulbs are already popular because of their longer life e.g. 30000 hours, power efficiency etc. Now smart LED light bulbs e.g. STACK bulb shown in Figure 34, is equipped with sensors and can react with ambient light, room occupancy etc. The bulb can turn itself off when the last person leaves the room or at sunrise, mimic daylight throughout the day etc. It also learns the lighting behaviour or preferences of the user and can reproduce the habit the while user is away e.g. holiday [49]. This can improve the security of the house against burglary.

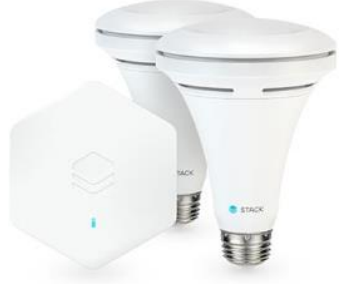

Figure 34: STACK down light starter kit [49].

\section{Miscellaneous}

\subsection{Personal Assistant}

Leading mobile device manufactures have introduced "Personal Assistant" e.g. Apple Siri, Google Now, Microsoft Cortana etc. as shown in Figure 35. These software tools use natural language user interface to interact with the user. The personal assistants can perform various tasks e.g. making phone calls, sending messages, scheduling meetings, 
launching browser, Internet searching, getting updated traffic information, obtaining weather forecast, answering questions etc.

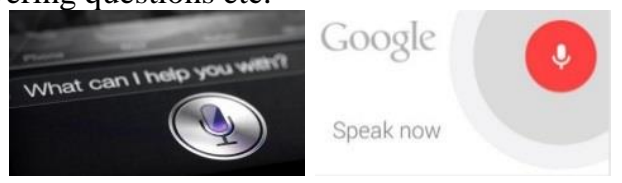

Figure 35: The Apple Siri [50] and the Google Now [51].

\subsection{Baby Monitors}

A number of smart devices are available to monitor baby activities as shown in Figure 36. For example, the Sensible Baby SmartOne monitor [52] and the Owlet Vitals Monitor [53] etc. allow parents to monitor baby's position, movement, body temperature, heart rate, oxygen level etc. on a smartphone or tablet in real time.

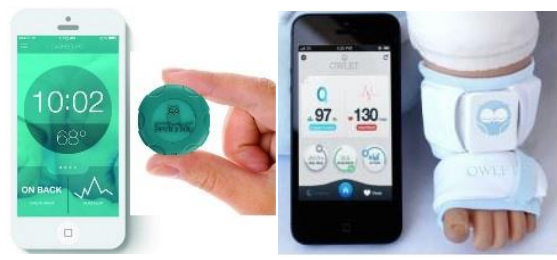

Figure 36: The Sensible Baby SmartOne monitor [52] and the Owlet Vitals Monitor [53].

\subsection{Real Time Natural Language Translation Tools}

Many smartphones are able to translate one language into another using translation tool. Recently Google has developed a real time translation tool based on images [54] which is shown in Figure 37 and supports a number of languages.

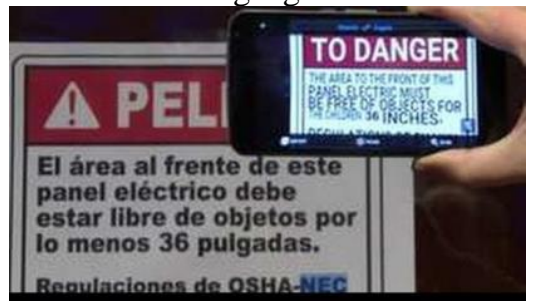

Figure 37: Real time natural language translation on a smartphone [54].

\subsection{Habit Changing Wristband}

Technology can help us to get rid of bad habit as well. For example, the Pavlok, shown in Figure 38, can be programmed e.g. visiting time-wasting websites, launching a maximum number of tabs in the browser etc. and it will generate an electric shock for the user to remind of bad habit [55].
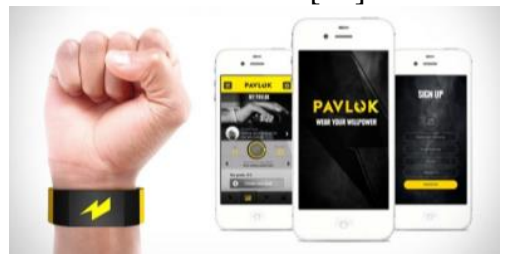

Figure 38: The Pavlok wristband [55].

\section{Conclusion}

Latest HCI innovations have made many technologies e.g. virtual reality, personal digital assistant, biometric authentication e.g. finger print scanner etc. available to us and have made our lives convenient, secure etc. Nowadays, we can monitor our health, manage our daily schedules, navigate from one place to another etc. using different forms of hardware and software HCIs. In the desktop computing HCI, many areas e.g. real time translation of one natural language into another, object recognition from images, low cost virtual studio, 3D scanner to produce 3D printed models etc. can be identified as the future researches. On the other hand, for mobile computing, e.g. eye movement tracking, hand gesture recognition, obtaining heart rate by putting a finger on the touchscreen for some time, starting a vehicle remotely, feedback on driving behaviour, augmented reality headset for vehicle e.g. engine maintenance, fully autonomous car etc. can be identified as future researches.

\section{References}

[1] "Curved Monitors - Pros and Cons | Ebuyer Blog," Ebuyer, 05Jan-2015. [Online]. Available:

http://www.ebuyer.com/blog/2015/01/curved-monitors-pros-andcons/. [Accessed: 01-Apr-2015].

[2] "Infographic: High End Display PPI Showdown | Infogram," 21Oct-2013. [Online]. Available: https://infogr.am/High-EndDisplay-PPI-Showdown. [Accessed: 12-Apr-2015].

[3] "Galaxy S6 \& Galaxy S6 edge - Pre-order online." [Online]. Available: http://www.samsung.com/uk/galaxys6/. [Accessed: 02-Apr-2015].

[4] S. Anthony, "LG's flexible and transparent OLED displays are the beginning of the e-paper revolution," ExtremeTech. [Online]. Available: http://www.extremetech.com/computing/186241-lgsflexible-and-transparent-oled-displays-are-the-beginning-of-thee-paper-revolution. [Accessed: 03-Apr-2015].

[5] "The smallest things make the biggest impression," Celluon. [Online]. Available:

http://www.celluon.com/products_epic_overview.php. [Accessed: 14-Jan-2016].

[6] "The VK200 KEYFOB® Virtual Keyboard," CTX Virtual Technologies. [Online]. Available:

http://www.ctxtechnologies.com/products/vk-200-keyfobvirtual-keyboard/. [Accessed: 14-Jan-2016].

[7] "Onecue replaces all your remote controls with hand gestures." [Online]. Available: http://www.gizmag.com/onecue-gesturecontrol/34902/. [Accessed: 19-Jan-2016].

[8] "How to Catch Brain Waves in a Net," IEEE Spectrum, 21-Aug2014. [Online]. Available:

http://spectrum.ieee.org/biomedical/bionics/how-to-catch-brainwaves-in-a-net. [Accessed: 01-Apr-2015].

[9] "Look, no hands (or feet): Scientists develop car that can be driven just by THINKING," Mail Online. [Online]. Available: http://www.dailymail.co.uk/sciencetech/article-

1359512/Computer-scientists-Germany-invented-car-steeredpower-thought.html. [Accessed: 01-Apr-2015].

[10] "Emotiv EPOC / EPOC+," Emotiv. [Online]. Available: http://emotiv.com/epoc.php. [Accessed: 02-Apr-2015].

[11] "Emotiv Claims Its Brainwave Scanner Allows People to Control Wheelchairs with Their Minds," Wearable Tech World, 03-Feb2014. [Online]. Available:

http://www.wearabletechworld.com/topics/from-theexperts/articles/369076-emotiv-claims-its-brainwave-scannerallows-people-control.htm. [Accessed: 01-Apr-2015].

[12] “Microsoft HoloLens," Microsoft HoloLens. [Online]. Available: http://www.microsoft.com/microsoft-hololens/en-us. [Accessed: 01-Apr-2015].

[13] "The best VR headsets," Wareable, 06-Mar-2015. [Online]. Available: http://www.wareable.com/headgear/the-best-ar-andvr-headsets. [Accessed: 01-Apr-2015]. 
[14] "Rift," Oculus VR. [Online]. Available: https://www.oculus.com/rift/. [Accessed: 01-Apr-2015].

[15] R. Preston, "“Virtual mannequins' promise better fit for online shoppers," BBC News. [Online]. Available: http://www.bbc.co.uk/news/technology-25812130. [Accessed 12-Apr-2015].

[16] "Virtual Fitting Rooms - are they a gimmick or an essential too for fashion ecommerce?| App Commerce Platform," Poq Studio | App Commerce Platform. [Online]. Available: http://poqstudio.com/2013/02/virtual-fitting-rooms-are-they-agimmick-or-an-essential-tool-for-fashion-ecommerce/. [Accessed: 12-Apr-2015].

[17] "Virtual Fitting Room Courtesy of the Kinect," Daily Bits. [Online]. Available: http://www.dailybits.com/virtual-fittingroom-courtesy-of-kinect/. [Accessed: 12-Apr-2015].

[18] "Google Cardboard, Experience virtual reality in a simple, fun, and inexpensive way," Google. [Online]. Available: https://www.google.com/get/cardboard/index.html. [Accessed 03-Apr-2015].

[19] "Valve reveals haptic game controller for release in 2014," BBC News, 27-Sep-2013. [Online]. Available: http://www.bbc.co.uk/news/technology-24304272. [Accessed: 01-Apr-2015].

[20] "Products | Tactical Haptics," Tactical Haptics. [Online]. Available: http://tacticalhaptics.com/products/. [Accessed: 01Apr-2015].

[21] "Google Glass UK release date, price and specs: Google Glas will go offsale on 19 January; how to buy Google Glass," PC Advisor. [Online]. Available:

http://www.pcadvisor.co.uk/features/gadget/3436249/googleglass-release-date-uk-price-specs/. [Accessed: 03-Apr-2015].

[22] “Apple (United Kingdom) - Apple Watch," Apple. [Online]. Available: https://www.apple.com/uk/watch/. [Accessed: 02Apr-2015].

[23] "Samsung Galaxy Gear Watch (Jet Black) - 1.63" Super AMOLED, 1.9MP, 4GB," Samsung UK. [Online]. Available: http://fb.uk.samsung.com/consumer/mobiledevices/wearables/gear/SM-V7000ZKABTU. [Accessed: 02Apr-2015].

[24] B. Kenney, "The Best Standalone Smartwatch Options Available," SmartWatches.org, 17-Oct-2014. [Online]. Available: http://smartwatches.org/learn/best-standalonesmartwatch-options/. [Accessed: 19-Jan-2016].

[25] S. Hill, "Smart jewelry is proving wearable tech doesn't have to be hideous," Digital Trends. [Online]. Available:

http://www.digitaltrends.com/mobile/smart-jewelry-roundup/. [Accessed: 03-Apr-2015].

[26] P. Rincon, "Smart shoe devices generate power from walking," BBC News. [Online]. Available:

http://www.bbc.co.uk/news/science-environment-30816255. [Accessed: 05-Apr-2015].

[27] "Watch your step -- with a Bluetooth-connected insole," CNET [Online]. Available: http://www.cnet.com/uk/news/watch-yourstep-with-a-bluetooth-connected-insole/. [Accessed: 06-Apr2015].

[28] L. Kelion, "CES 2015: Preview of the new tech on show in Las Vegas," BBC News. [Online]. Available: http://www.bbc.co.uk/news/technology-30643396. [Accessed: 06-Apr-2015].

[29] "Sensoria Fitness Smart Sock Preview," CNET. [Online]. Available: http://www.cnet.com/uk/products/sensoria-fitnesssmart-sock/. [Accessed: 06-Apr-2015].

[30] "Best fitness trackers 2015: Jawbone, Misfit, Fitbit, Garmin and more," Wareable. [Online]. Available: http://www.wareable.com/fitness-trackers/the-best-fitnesstracker. [Accessed: 03-Apr-2015]

[31] "FITGuard: Alerts Athletes Of Potential Head Injuries NXTInsight." [Online]. Available: http://nxtinsight.com/fitguardalerts-athletes-injuries/. [Accessed: 16-Jan-2016]

[32] "M-Tracer ${ }^{\mathrm{TM}}$ Golf Swing Analyzer," Epson. [Online]. Available http://www.epson.com/cgi-bin/Store/jsp/Landing/m-tracer-golfswing-analyzer.do?ref=van_golf. [Accessed: 16-Jan-2016]

[33] “Inside Google X's Smart Contact Lens," Re/code. [Online]. Available: http://recode.net/2014/01/16/inside-google-xs-smartcontact-lens/. [Accessed: 03-Apr-2015]

[34] "Google unveils 'smart contact lens' to measure glucose levels,' $B B C$ News. [Online]. Available: http://www.bbc.co.uk/news/technology-25771907. [Accessed: 03-Apr-2015]
[35] "Augmented Reality in a Contact Lens," IEEE Spectrum [Online]. Available: http://spectrum.ieee.org/biomedical/bionics/augmented-realityin-a-contact-lens/eyesb1. [Accessed: 03-Apr-2015].

[36] M. Roberts, "Bionic contact lens 'to project emails before eyes,"” BBC News. [Online]. Available:

http://www.bbc.co.uk/news/health-15817316. [Accessed: 03Apr-2015].

[37] L. Kelion, "Fingerprint sensor revealed by Qualcomm at MWC," $B B C$ News. [Online]. Available:

http://www.bbc.co.uk/news/technology-31692988. [Accessed: 07-Apr-2015]

[38] T. Perry, "A Temporary Tattoo That Senses Through Your Skin," 29-May-2015. [Online]. Available: http://spectrum.ieee.org/biomedical/devices/a-temporary-tattoothat-senses-through-your-skin. [Accessed: 15-Jan-2016].

[39] "It would be really great to have a closed-loop artificial pancreas device (APD) system on the market...it feels like we have been waiting for this for forever!," Integrated Diabetis Services, 14Jan-2016. [Online]. Available: http://integrateddiabetes.com/closed-loop-artificial-pancreassystem-update/.

[40] "NEJM: First thought-controlled prosthetic leg," MedCity News. [Online]. Available: http://medcitynews.com/2013/09/nejm-firstthought-controlled-prosthetic-leg/. [Accessed: 02-Apr-2015].

[41] J. Gallagher, "Brain-reading implant controls arm," BBC News, 22-May-2015. [Online]. Available: http://www.bbc.co.uk/news/health-32784534. [Accessed: 17Jun-2015].

[42] E. Ackerman, "Tesla Model S: Summer Software Update Will Enable Autonomous Driving," 23-Mar-2015. [Online]. Available: http://spectrum.ieee.org/cars-thatthink/transportation/self-driving/tesla-model-s-to-combinesafety-sensors-to-go-autonomous. [Accessed: 19-Jan-2016]

[43] "Navdy feels like driving in the future," Navdy. [Online]. Available: https://navdy.tilt.com/start-driving-in-the-future. [Accessed: 15-Jan-2016]

[44] "Augment reality in the car, road projection on the windshield HUD Head-Up Display." [Online]. Available: http://hudwayapp.com/. [Accessed: 15-Jan-2016].

[45] "D-air ${ }^{\circledR}$ Racing," Dainese. [Online]. Available: http://www.dainese.com/en_en/d-air/racing/. [Accessed: 16-Jan2016].

[46] L. Smith, "Ford unveils a car seat which detects when a driver is having heart attack," The Independent, 20-Oct-2014. [Online]. Available: http://www.independent.co.uk/news/uk/homenews/ford-unveils-a-car-seat-which-detects-when-a-driver-ishaving-heart-attack-9807042.html. [Accessed: 07-Apr-2015].

[47] "Using Confused.com MotorMate," 07-Oct-2013. [Online]. Available: http://www.honestjohn.co.uk/news/tax-insurance-andwarranties/2013-02/blog-using-confusedcom-motormate/ [Accessed: 15-Jan-2016]

[48] G. Kelly, "Smart thermostats reviewed: Which can save you the most?," The Guardian, 27-Jan-2015.

[49] "Stack Downlight Starter Kit," store.stacklighting.com. [Online]. Available: http://store.stacklighting.com//ProductDetails.asp?ProductCode= BR30KIT. [Accessed: 16-Jan-2016].

[50] "Siri, Your wish is its command," Apple. [Online]. Available: https://www.apple.com/uk/ios/siri/. [Accessed: 02-Apr-2015]

[51] "Google Now. The right information at just the right time.," Google. [Online]. Available: https://www.google.co.uk/landing/now/. [Accessed: 02-Apr2015].

[52] "No more tiptoeing around," Sensible Baby. [Online]. Available: http://mysensiblebaby.com/. [Accessed: 06-Apr-2015].

[53] "Owlet Vitals Monitor," Thing Alive. [Online]. Available: http://thingalive.com/owlet-vitals-monitor. [Accessed: 06-Apr2015].

[54] "Pardon? Testing Google's speedy translation tool," BBC News. [Online]. Available: http://www.bbc.co.uk/news/technology30824033. [Accessed: 07-Apr-2015].

[55] "Pavlok Uses Mild Electric Shock To Help You Break Any Habit," Pavlok. [Online]. Available: http://pavlok.com/ [Accessed: 06-Apr-2015]. 


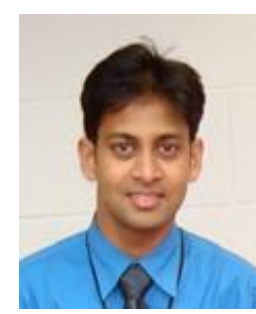

Mohammad S. Hasan received his BSc and first MSc in Computer Science. He obtained his second MSc in Computer and Network Engineering from Sheffield Hallam University, UK. He has been awarded a PhD at Staffordshire University, UK in Networked Control Systems over Mobile Ad-hoc Network (MANET). Currently he is a Senior Lecturer and a member of the Mobile Fusion (MF) Applied Research Centre (ARC) at Staffordshire University. His research interests include computer networks, networked control systems, remotely controllable mobile robot systems, real time systems, wireless sensor networks etc. Email: m.s.hasan@staffs.ac.uk

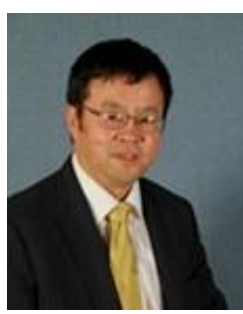

Hongnian Yu is currently a Professor of Computer Science at Bournemouth University. He has extensive research experience in modelling and control of robots and mechatronics devices and neural networks, mobile computing, modelling, scheduling, planning and simulations of large discrete event dynamic systems, RFID with applications to manufacturing systems, supply chains, transportation networks and computer networks. He has published over 200 research papers and held several grants from EPSRC, the Royal Society, and other funding bodies. He is a member of the EPSRC Peer Review College and serves on various conferences and academic societies. Email: yuh@bournemouth.ac.uk 